\title{
A Belief Rule Based Expert System for Datacenter PUE Prediction under Uncertainty
}

\author{
Mohammad Shahadat Hossain, Member, IEEE, Saifur Rahaman, Ah-Lian Kor, Member, IEEE, Karl \\ Andersson, Senior Member, IEEE, and Colin Pattinson
}

\begin{abstract}
A rapidly emerging trend in the IT landscape is the uptake of large-scale datacenters moving storage and data processing to providers located far away from the end-users or locally deployed servers. For these large-scale datacenters power efficiency is a key metric, with the PUE (Power Usage Effectiveness) and DCiE (Data Centre infrastructure Efficiency) being important examples. This article proposes a belief rule based expert system to predict datacenter PUE under uncertainty. The system has been evaluated using real-world data from a data center in the UK. The results would help planning construction of new datacenters and the redesign of existing datacenters making them more power efficient leading to a more sustainable computing environment. In addition, an optimal learning model for the BRBES demonstrated which has been compared with ANN and Genetic Algorithm; and the results are promising.
\end{abstract}

Index Terms-Predictive Modeling, Datacenter, Energy Efficiency, Belief Rule Based Expert System.

\section{INTRODUCTION}

$\mathrm{T}$ HE raw data used for this paper is sourced through the project entitled Measuring Data Centre Efficiency funded under the JISC Greening ICT initiative, call for projects 14/10 of October 2010 [1] [2]. The project provided real experimental data relating to the accurate measurements of energy consumption in different parts of the datacenter. Power Usage Effectiveness (PUE) is calculated based on the collated data and is used to provide trends, and prediction of the data center efficiency.

According to the US datacenter energy usage report [3], in 2014, the total energy consumption of US datacenters was estimated to be 70 billion kWh (i.e. $1.8 \%$ of total US electricity consumption). US data center energy use is expected to increase by $4 \%$ from $2014-2020$ and based on current estimates, the projected consumption is approximately 73 billion kWh in 2020.

As discussed in [2], it is imperative that data center energy efficiency be prioritized in order to help reduce its impact on the environment and climate change. Microsoft Corporation [5] 10 best practices views the data center as an ecosystem comprising IT systems (e.g. servers, storage, network, etc.), mechanical systems (e.g. chillers, cooling systems, pumps, etc.), and electrical systems (internal trans-

- Mohammad Shahadat Hossain is with the Department of Computer Science and Engineering, University of Chittagong, University-4331, Bangladesh.

E-mail: hossain_ms@cu.ac.bd

- Saifur Rahaman is with the Department of Computer Science and Engineering, International Islamic University Chittagong, Chittagong-4203, Bangladesh.E-mail: saifurcse@iiuc.ac.bd

- Ah-Lian Kor and Colin Pattinson are with School of Computing, Creative Technologies and Engineering, Leeds Beckett University, 101, Caedmon Hall, Headingley Campus, Leeds LS6 3QS, United Kingdom. E-mail: a.kor@leedsbeckett.ac.uk and c.pattinson@leedsbeckett.ac.uk

- Karl Andersson is with Pervasive and Mobile Computing Laboratory, Luleå University of Technology, SE-931 87 Skellefteå, Sweden. E-mail: karl.andersson@ltu.se

Manuscript received December 30, 2016; revised MMM DDD, $20 Y$ Y. formers, UPS, etc.) which facilitates environmental controls, monitoring, and networking. The only effective means to measure the efficiency of data center operations is to take a holistic approach (IBM Corporation, 2012) that considers the measurements (and trade-offs) of all components within the ecosystem. Data Center IT equipment are: servers, storage, and networks [3]. To reiterate, the dataset for this paper is obtained from the JISC funded project Measuring Data Center Efficiency [1] [2]. The aim of the project was to monitor the energy consumption in different parts of the data center with varying metering topology. The primary focus was the temperature parameter and an investigation of its effect on data centre energy consumption improvement using PUE. Other specific objectives of the project encompassed the following:

1) identify appropriate power consumption reduction measures which can best be implement in the data centre;

2) identify appropriate candidate locations for installation - identify the locations and applications which are used as test beds, focusing particularly on the main university data centre including server room equipment, UPS and environmental control systems;

3) determine suitable metrics and measurement methods - review the range of possible measurement metrics and metering approaches, selecting those which best allow us to evaluate the relative performance of selected systems against the project requirements;

4) install required power metering and monitoring devices ensure that the measurement and monitoring facilities required are in place. This involves integrating the new metering devices into existing software systems used for collecting the data and producing meaningful management reports; 
5) record data centre power consumption, calculate PUE only at campus/building level power consumption data. This data is gathered over a period of at least two months of typical of business activity.

The findings from the JISC funded project are based on both trend and comparatively analyses. However, this paper aims to extend this work by incorporating the use of a belief ruled based expert system for the prediction of a data center PUE with uncertainty. It ought to be noted that energy-related security in the context of a data centre [4] is not the focus of this study, but our future work might cover those aspects at a later stage.

This remainder of this article is organized according in the following way: Section 2 covers a literature review, while Section 3 contains an overview of Belief Rule Based Expert Systems. Then, Section 4 introduces how the proposed Belief Rule Based Expert System is used to predict PUE, while Section 5 discusses the results. Finally, Section 6 concludes our findings and indicates our future work.

\section{Related Work}

$\mathrm{T}$ HE data center industry is very energy intensive [6], and in the year 2015, 416.2 terawatt hours of electricity was consumed by the worlds data centers [7]. In the US alone, the data centers used approximately 91 billion kilowatt hours electricity [8] which accounts to about 1.8 percent of total US electricity consumption [9]. It is estimated that by 2020, the demand will increase to 139 billion kilowatt hours (which is equivalent to approximately 53\% increase the electricity output of 51 power plants, each capable of generating 500 megawatts) [8]. The increase in the amount of energy consumed is due to the transmission, processing, and storage of billions of gigabytes of information relating to IoTs, clouds, etc. [9]. Data center energy efficiency will help lower costs and environmental impact [10] and it could help save 620 billions kilowatt hours of energy in the US alone (equivalent to USD 60 billions) [9]. In order to better understand data center energy efficiency, we have conducted a critical literature survey on energy efficiency best practices, energy efficiency metrics and predictive modelling for energy consumption in data centers.

\subsection{Data Center Energy Efficiency Best Practices}

Data center energy efficiency best practices could be coded into the following categories: IT systems energy efficiency; data center design and location; electrical systems; and air management and cooling systems.

\subsubsection{IT Systems Energy Efficiency}

\subsubsection{Energy Efficient Servers}

A majority of data center servers are underutilized where most of the time, they perform below $20 \%$ of their potential and yet consuming full power in the process [11]. According to Energy Star, their certified servers would have the following energy efficient features: servers with variable speed cooling fans [ibid]; CPU speed throttling and power saving technology to reduce processors clock rate and voltage when the processor is idle [12] [13]. Energy Star claimed

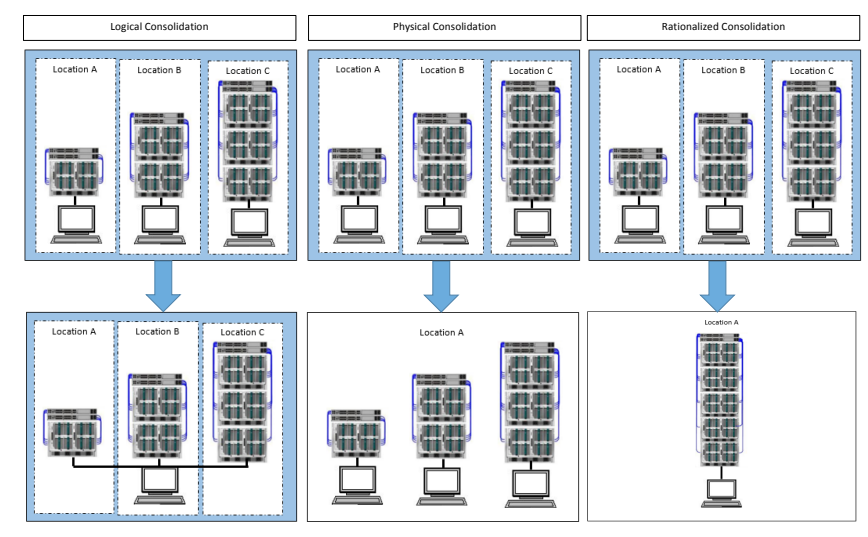

Fig. 1. Types of Consolidation (according to [17])

that servers that meet their requirements would be $30 \%$ more efficient than standard servers [11]. The savings made for a single Energy Star-qualified servers is expected to be 600 kilowatt hours (at 50\% utilization) and 1200 kilowatt hours (at idle) annually or 2400-4800 kilowatt hours over the useful life of a server (typically 4 years) [11] [12].

\subsubsection{Consolidation}

Gartner [14] discusses the best practices in server consolidation and views IT infrastructure consolidation as the main focus of IT infrastructure and operations cost reduction initiatives. Server consolidation is an approach to the efficient usage of computer server resources because it helps reduce the total number of servers by placing more applications on fewer machines so as to reduce underutilization of the servers [11] [13] [15] [16], maximise the efficient use of space [15], and reduce hardware and operating costs [16]. There are three categories of server consolidation (see Figures 1 and 2):

1) Logical consolidation - all servers are placed under the management of a single centralized group [14] and the goal is to implement common processes as well as enable standard systems management procedures across the server applications [17];

2) Physical consolidation - it is synonymous to data center consolidation [14] which involves the colocation of multiple platforms at fewer locations [17] and thus the reduction of the number of data centers where the servers are located. According to Microsoft [18], datacenter consolidation will bring about IT consolidation which will help enhance IT infrastructure, reduce the data center footprint, power and cooling costs as well as better control and manage both physical and virtual machine sprawl;

3) Rationalized consolidation - this involves server and other IT infrastructure rationalization [14]. This technique entails the implementation of multiple applications over less but more powerful platforms through workload management and partitioning [17]. 


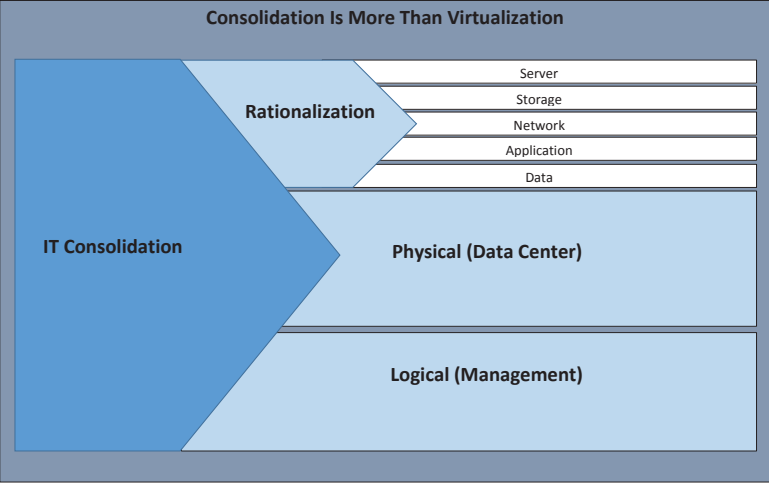

Fig. 2. Types of Consolidation (according to [14]

In summary, the advantages of consolidation are:

1) optimization of investment in infrastructure through the reduction of the number of servers necessary to support the running of applications [19], and maximize return on investment (ROI) [20];

2) yield large TCO savings [14];

3) reduce costs for server hardware and software [19]. According to IBM [ibid] typical savings made are hardware costs reduced $33 \%$ to $70 \%$, maintenance costs reduced up to $50 \%$, support costs reduced by as much as $33 \%$, floor space and facility costs reduced 33 to $50 \%$. VMWare estimates the total energy savings to be $80 \%$ [16];

4) bring about business and IT agility [21].

\subsubsection{Virtualization}

Virtualization is a method for consolidation [22] and is viewed as a fundamental element of a green data center [23] which could change data center dynamics [24]. It is a method for concurrently running multiple virtual machines on a single physical host [11] [25]. It is a way to consolidate servers so that the different multiple workloads could be run on one physical host server [13] through the deployment of a hypervisor (i.e. a software capable of hosting multiple virtual machines) [22]. Thus, this swaps a number of underutilized servers for a single highly utilized server with concurrent running of multiple virtual machines [26]. Cisco uses VMWare Infrastructure 3 [ibid] [26] as the foundation for server virtualization in the data center an it could create virtualized servers, each with multiple CPUs and multiple gigabytes of memory. The advantages of the virtualization technology [24] [25] [26] [27] are:

1) reduce costs through reduced complexity, enhanced resource utilization, reduced use of floor space, and more efficient power as well as cooling;

2) improve service through enhanced performance and optimized scalability, enhanced service levels;

3) better managed risks through improved uptime, availability and increased recoverability;

4) yields a rewarding return on investment (ROI) [28].
Consequently, virtualization technologies within a data center is expected to reduce energy consumption by $80 \%$ and every virtualized server is estimated to save 7000 kilowatt hours of electricity and four tons of carbon dioxide emissions annually [23]. Additionally, the IT infrastructure has become more dynamic, flexible, agile, and responsive to fluctuating capacity demands [ibid] [24] [25] by detaching workloads and data from the functional side of the physical infrastructure [28]. However, a large scale of deployment of virtual machines could result in a stress on the data center IT infrastructure if the traditional server model is still in use [24]. In order to appropriately address this issue, the new design requirements of the data center will have to be looked into [ibid] and at the same time, conside the scalability of the right-sized power and cooling systems [29].

\subsubsection{Data Center Design and Location}

The capacity design of a data center is important because the energy efficiency of the data center will be directly impacted by the actual proportion of the design load being implemented [30]. Thus, the lower the actual load utilization compared to its design maximum, the lower would be the efficiency which would have a negative impact on the data center overall energy efficiency. Power density reveals how much IT equipment could be placed in a rack and a lower power density implies the use of more racks for the IT equipment [ibid]. However, the specification of operating power density is challenging. A given example [31] is that specification of traditional densities of 430-861 Watts $/ \mathrm{m}^{2}$ will result in the challenge of deploying the latest IT technologies while a power density specification of 6,458-10,764 Watts $/ \mathrm{m}^{2}$ for latest generation IT equipment will put additional stress to the data center cooling systems. APC [ibid] has detailed an improved method for specifying data center power intensity in order to address the following issues: assurance of compatibility with high density IT equipment; reduction of waste relating to electricity, space, and capital expenses; validation of IT deployment plans with cooling and power capability.

The geographical location of a data center will have implications on the cost of power, access to renewable energy, and availability of free cooling air [30]. Currently, the American Society of Heating, Refrigeration and Air-Conditioning Engineers (ASHRAE) recommends temperatures of up to $27^{\circ} \mathrm{C}$ (up $34^{\circ} \mathrm{C}$ for some IT equipment) and consequently, free air-cooling is reconsidered [32]. However, at the same time, the humidity of the air would have to be carefully weighed due to its negative impacts on the operational conditions and lifespan of the equipment. This means even though the temperature of the incoming air is within the recommended range, with its humidity controlled using an air economizer and also filtered so as to capture particulates that could cause problems to the data centers [ibid]. Details of the recommendations for inlet air conditions are found in Section 2.1.4.

According to US Department of Energy $[11]^{6}$, it is recommended that the power systems within a data center exist as co-generation systems. This means that power generators with data centers (e.g. diesel generators that are used as sources of backup power) could be utilized as co-generation systems where waste heat produced by the generator could 
be captured to run an absorption chiller to cool the data center. Energy recovery from the generator and waste heat from cooling air could be incorporated into the design of new data centers [11] [33]. This is made possible through the use of heat recovery chillers for the recovery and reuse of heat from data center equipment environments for comfort heating of office buildings.

\subsubsection{Electrical Systems}

In order to reduce data center energy inefficiency, it is recommended that more energy efficient UPSs and PDUs be used [13]. According to Energy Star [ibid], a power management system could reduce switching energy losses in inverters as well as transformers and an Energy Star certified UPS could cut energy losses by $30-55 \%$. Thus, it is vital to increase the UPS itself and also through lower heat loads as well as reduced transformer losses [11]. Metered PDUs could provide information on workloads within a data center and it is recommended that PDUs be located centrally within the data center so as reduce power lost in the form of heat [ibid]. In order to reduce waste heat, PDUs could use high-efficiency transformers that are 2-3\% more efficient [13], have parameters of the transformer specified in such a manner that energy efficiency could be optimized (e.g. dry-type transformer with a $176^{\circ} \mathrm{F}$ temperature rise consumes $13 \%$ to $21 \%$ less energy than a $302^{\circ} \mathrm{F}$ rise unit), or reduce the number of installed PDUs with in-built stepdown transformers $[11]^{6}$.

Power sourced to a data center could either be threephase or single-phase. It is argued that the three-phase machines cum controls could be smaller, lighter in weight and more efficient while the amount of copper wire required for three-phase power distribution will be reduced by $25 \%$ compared to single-phase power distribution [34] [35], the primary voltage schemes (three-phase) available in the world are: North American 480/208/120 V (600/208/120 in Canada) or the $400 / 230 \mathrm{~V}$ system in used Europe and some parts of Asia [35]. One source of electrical power loss for both AC and DC distribution is stepping down the inbound voltage (usually $12 \mathrm{kV}$ AC or more) through the three-phase to the operating voltage of the devices within the data center (usually 100V 240V) [11]. Some data centers are exploring the use of DC (direct current) to improve energy efficiency, however, AC (alternating current) is still predominantly used in data centers [35]. Some of the recommendations [11] for energy efficient electrical power distribution within the data center are: minimize the resistance of the distribution channels; maintain a higher voltage to reduce current; use switch-mode transistors for power conditioning; place all voltage regulators close to the load so as to minimize distribution losses.

\subsubsection{Air Management and Cooling Systems}

The Center of Expertise for Energy Efficiency in Data Centers [36] has provided an air management tool which contains air management recommendations relating to: supply airflow rate and inlet air temperature without an impact on the temperature of the environment the IT equipment is operating in; associated savings for fans and chillers; raised floor cooling with hot/cold aisles, etc.
TABLE 1

ASHRAE Recommended and Allowable Inlet Air Conditions for Class 1 and 2 Data Centers [37]

\begin{tabular}{|c|c|c|c|}
\hline & $\begin{array}{c}\text { Class 1 and } \\
\text { Class 2 } \\
\text { Recommended } \\
\text { Range }\end{array}$ & $\begin{array}{c}\text { Class 1 } \\
\text { Allowable } \\
\text { Range }\end{array}$ & $\begin{array}{c}\text { Class 2 } \\
\text { Allowable } \\
\text { Range }\end{array}$ \\
\hline Low Temp. Limit & $18^{\circ} \mathrm{C} \mathrm{DB}$ & $15^{\circ} \mathrm{C} \mathrm{DB}$ & $10^{\circ} \mathrm{C} \mathrm{DB}$ \\
\hline High Temp. Limit & $27^{\circ} \mathrm{C} \mathrm{DB}$ & $32^{\circ} \mathrm{C} \mathrm{DB}$ & $35^{\circ} \mathrm{C} \mathrm{DB}$ \\
\hline Low Moist. Limit & $5.5^{\circ} \mathrm{C} \mathrm{DP}$ & $20 \% \mathrm{RH}$ & $20 \% \mathrm{RH}$ \\
\hline High Moist. Limit & $6 \%^{\circ} \mathrm{RH} \&$ & $80 \% \mathrm{RH} \&$ & $80 \% \mathrm{RH} \&$ \\
& $15^{\circ} \mathrm{C} \mathrm{DP}$ & $17^{\circ} \mathrm{C} \mathrm{DP}$ & $21^{\circ} \mathrm{C} \mathrm{DP}$ \\
\hline
\end{tabular}

\subsubsection{Inlet Air and Humidity}

The American Society of Heating, Refrigerating and AirConditioning Engineers (ASHRAE) or Network Equipment Building System (NEBS) have outlined the recommended and allowable environmental envelop for inlet air entering IT equipment within a data center (see Table 1) [37]. This recommended envelop provides a guideline on energy-efficient operations with high reliability. If the inlet air conditions are above the recommended range then internal fans in the servers will operate at higher speeds thus resulting in a higher energy consumption [ibid].

Research has been investigated on the effect of temperature on energy efficiency [1] [2] [61]. According to Patterson [61], increasing the ambient temperature within a data center could result in higher power usage of some components and systems within the data center. The research that he conducted reveals that there is an optimum temperature for the data center operations that is dependent on the behaviour of the IT equipment, cooling system, location (i.e. external temperature, etc.).

\subsubsection{Variable Fan Speeds}

According to Energy Star [38], the energy consumed by Computer Room Air Conditioning (CRAC) and Computer Room Air Handling (CRAH) unit fans are estimated to account for $5-10 \%$ of a data center's total energy usage. However, the speed of these fans cannot vary with data center server load. Consequently, the US Department of Energy [39] has documented a case study on three retrofits of variable speed controls to existing constant-speed fans in computer-room air conditioners (CRACs) within three data centers. This case study demonstrates that such retrofit of variable speed operation is possible and it could bring about significant energy savings accompanied with comparable or improved cooling and reliability. The estimated savings for a variable fan speed are: a reduction of $10 \%$ in fan speed reduces the fan's energy use of approximately $25 \%$ [38]; a reduction of cooling system energy use of $22-32 \%$ when compared to constant speed fan [39].

\subsubsection{Hot/Cold Aisles Layout with Containment}

According to Energy Star, hot/cold aisle layout will reduce cooling costs due to better management of airflow [61] and the estimated fan energy savings is between $20-25 \%$. However, when accompanied with containment systems 


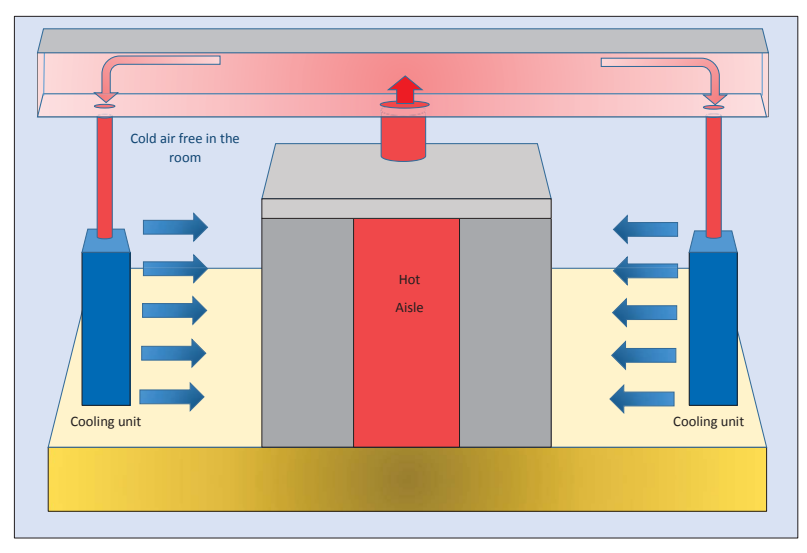

Fig. 3. Hot/cold aisle with containment systems [41]

(see Figure 3) which are physical barriers used to prevent the mixing of cold inlet air and hot exhaust air, it delivers further $20 \%$ of energy saving for the chillers [40]. According to Schneider Electric [41], a hot aisle is enclosed within a containment system so as to capture hot exhaust air from the IT equipment and leave the rest of the room to be filled with cold air. This will help separate the hot and cold air streams are separated.

In summary, the trend towards the emergence of data center infrastructure management (DCIM) sees the convergence of IT and building facilities functions within an organization in order to provide a holistic view of a data center to bring about efficiency in terms of energy, performance, equipment and use of floor space [42]. The US Department of Energy has provided recommendations for energy efficient data center design [11] [43] accompanied by a data center master list of efficiency actions and technologies to support data center efficiency [42]. In Europe, the EU Code of Conduct on Data Centres" Energy Efficiency [44] is a voluntary initiative aimed at reducing the environmental, economic, and energy-supply security impact of data centres and Europe standardization for green data centers [45]. Energy Star has listed 10 ways to save energy in a data center [46], Microsoft outlines 7 tenets of data center energy efficiency [47], while Google has provided a list of best practices [48]. Microsoft has developed a data center sustainability strategy [49] while Energy Start has provided a case study on Google's green data centers [50].

\subsection{Data Center Energy Efficiency Metrics}

According to the Green Grid Association [51], power usage effectiveness (PUE) is the "industry-preferred metric for measuring infrastructure energy efficiency for data centers" which has been globally adopted. PUE "compares the amount of non-computing overhead energy (like cooling and power distribution) to the amount of energy used to power our actual machines" [52] it is used to reveal how well a data center total energy consumption scales with changes in its IT equipment loads [51]. PUE is the ratio of of total facilities energy to IT equipment energy while data center infrastructure efficiency (DCiE) is the inverse of PUE [ibid] [53].

\subsection{Predictive Modelling for Energy Consumption in Data Centers}

Predictive modelling in data centers will be considered as the "next frontier for condition maintenance" [54] and according to Koomey [55], predictive modelling in the context of a data center, could "unlock stranded capacity and identify practices for higher efficiency and reliability". Google estimates the use of AI for predictive modelling could reduce energy use within a data center by $15 \%$ [56]. Romonet has developed a set of Prognose software suite which could be employed to for predictive modelling of energy and capacity use within data centers [57]. This model uses PUE to determine energy efficiency of data centers. The Prognose simulator includes the following tools: add infrastructure devices, change parameters of devices, load IT devices, add additional information (e.g. energy tariff, climate data, etc.), monitoring tools to provide down to minute-granularity data whenever necessary, data storage facility (in a Prognose database). The acronymn DCPM (data center predictive modelling) has been ascribed this data center predictive modelling suite by Romonet [ibid] and the demonstration of the software capability has been discussed in [58] [59]. In brief, DCPM refers to the capability to forecast the performance of a data center in terms or energy consumption, energy efficiency, etc. [57]. However, the model employed for energy use prediction based on various parameter changes (e.g. change IT equipment load, fan speed controls of CRAH, and number of water-side economizers, etc.) will have to be run in successive iterations [58] [59]. Thermal News [54] provides a very coherent picture of three sets of intertwined variables whose tradeoffs contribute to the overall performance of a data center: IT availability (in terms of \% of load and failure); physical capacity (i.e. amount of design capacity that is available for use and scalability) and cooling efficiency (i.e. efficiency of cooling systems). On the other hand, Google has successfully run trials on the use of machine learning applications (using neural network) for data center optimization which takes into considerations the complex interaction of three primary systems within a data center: mechanical, electrical, and control [60]. Their results reveal that machine learning is an effective of leveraging existing sensor data to model a data center performance and improve energy efficiency. On the other hand, IBM has launched its new predictive modelling suite which primarily focuses on the business side of a data center: operational changes with growing business demands; cash flow analysis, physical threshold capacity; resiliency rationalization to support data center planning and management.

\section{Outline of Belief Rule Based expert SYSTEMS}

B ELIEF Rule Based Expert Systems (BRBES) are widely used to assess or evaluate the performance of a realworld system or to predict the occurrence of an event under uncertainty [63] [64]. The reason for this is that most of 
the real world systems are complex in nature due to the presence of infinite number of factors which are difficult to measure with $100 \%$ accuracy. BRBES consists of two components, namely knowledge-base and inference engine [65]. Belief rule base is used to represent uncertain knowledge whereas evidential reasoning is used to perform the task of inference or reasoning to generate decision scenarios from the BRBES [66]. The prediction of energy efficiency of data centre is also crucial since it involves many factors such as external temperature, server room temperature, humidity, fan speeds, speed of airflow, power density, etc. However, these factors cannot be measured with $100 \%$ accuracy.

Therefore, a BRBES is appropriate to predict energy efficiency of a data centre under uncertainty. Hence, this section presents an overview of the main components of the BRBES, focusing on the knowledge representation schema known as BRB and the inference procedures consisting of input transformation, rule activation weight calculation, belief degree update and rule aggregation. This will be followed by the presentation of the optimal learning procedures, where the gap between the output of the real system and the simulated system (in this case BRBES) will be demonstrated by adjusting the values of the various learning parameters.

\subsection{Domain Knowledge Representation in BRBES}

The Belief Rules are the extension of traditional IF-THEN rules. A belief rule consists of an antecedent part and a consequent part. The antecedent part consists of one or more than one antecedent attributes with associated referential values. While the consequent part consists of one consequent attribute associated with referential values and they are embedded with belief degrees. However, when the summation of the belief degrees is one then the rule is considered as complete otherwise it is incomplete. In this way, the issue of uncertainty due to incompleteness is addressed in the BRB. In addition, rule weights, antecedent attribute weight, and the belief degrees are considered as the learning parameters, which can be adjusted to obtain an optimal value from the BRBES as will be discussed later.

$$
R_{k}:\left\{\begin{array}{l}
\operatorname{IF}\left(P_{1} \text { is } A_{1}^{k}\right) \wedge\left(P_{2} \text { is } A_{2}^{k}\right) \wedge \ldots \wedge\left(P_{T_{k}} \text { is } A_{T_{k}}^{k}\right) \\
\operatorname{THEN}\left(C_{1}, \beta_{1 k}\right),\left(C_{2}, \beta_{2 k}\right), \ldots,\left(C_{N}, \beta_{N k}\right)
\end{array}\right.
$$

IF (External Temperature is Medium) and

(Room Temperature is High)

THEN PUE is (Very High, 0.0), (High, 0.7), (Medium, 0.1), (Low, 0.2), (Very Low, 0.0)

where

(Very High, 0.0), (High, 0.70), (Medium, 0.10), (Low, 0.20), (Very Low, 0.0) is a belief distribution associated with the referential values of the "PUE", which is the consequent attribute of the rule as shown in Equation 2. "Very High", "High", "Medium", "Low", and "Very Low" are the referential values of the consequent "PUE". The belief distribution states that the degree of belief associated with "High" is 70\%, 10\% degree of belief associated with

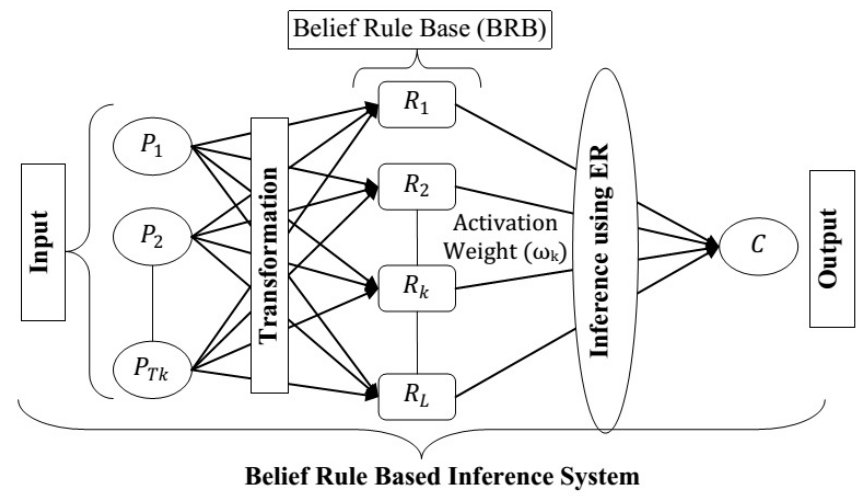

Fig. 4. Single-layer BRB inference architecture.

"Medium", while 20\% degree of belief is associated with "Low". In this belief rule, the total degree of belief is $(0.0+0.7+0.1+0.2+0.0)=1$; hence, the assessment is complete.

\subsection{BRB Inference Procedures}

The inference procedures consist of various steps including input transformation, rule activation weight calculation, belief degree update and rule aggregation using Evidential Reasoning approach. Figure 4 shows the structure of the inference procedures of the BRBES. These will be discussed accompanied with an example.

\subsubsection{Input Transformation and Rule Activation Weight Cal- culation}

The input transformation of a value of an antecedent attribute $P_{i}$ consists of distributing the value into belief degrees of different referential values of that antecedent. For example, if the value of antecedent attribute External Temperature of a data centre is $20^{\circ} \mathrm{C}$ then this needs to be transformed or distributed over its various referential values, consisting of Very High $(\mathrm{VH})$, High $(\mathrm{H})$, Medium (M), Low (L), and Very Low (VL). Through this distribution, each referential value will get a matching degree or degree of belief to demonstrate what amount of this input value $\left(20^{\circ} \mathrm{C}\right)$ will match with each of the referential values $(\mathrm{VH}$, H, M, L, VL) as shown in Equation 3.

$$
H\left(P_{i}, X\right)=\left(\mathrm{VH}, \alpha_{\mathrm{VH}}\right)\left(\mathrm{H}, \alpha_{\mathrm{H}}\right)\left(\mathrm{M}, \alpha_{\mathrm{M}}\right)\left(\mathrm{L}, \alpha_{\mathrm{L}}\right)\left(\mathrm{VL}, \alpha_{\mathrm{VL}}\right)
$$

When the matching degrees $\left(\alpha_{V H}, \alpha_{H}, \alpha_{M}, \alpha_{L}\right.$, and $\left.\alpha_{V L}\right)$ are assigned to the referential values of a rule then the rule is said to be activated. For example, in this case the rule represented by Equation 2. This phenomenon of rule activation is also called the packet antecedent of a rule. However, a rule consists of one or more than one antecedent attributes, which is the case with the rule represented by Equation 1. Therefore, it is necessary to find out the combined matching degree of a rule considering all the antecedent attributes. This can be achieved by applying a simple weighted multiplicative aggregation function as shown in Equation 4. The use of such multiplicative function demonstrates integration among the antecedent attributes of a rule [67] [68]. 


$$
\alpha_{k}=\prod_{i=1}^{T_{k}}\left(\alpha_{i}^{k}\right)^{\bar{\delta}_{k i}}
$$

where

$$
\bar{\delta}_{k i}=\frac{\delta_{k i}}{\max _{i=1 \ldots, T_{k}}\left(\delta_{k i}\right)} \text { is the relative weight of } P_{i} \text { used }
$$

in the $k$ th rule, which is calculated by dividing weight of $P_{i}$ with maximum weight of all the antecedent attributes of the $k$ th rule. By doing so, the value of $\delta_{k}$ becomes normalized, meaning that the range of its value should be between 0 and 1 . The combined matching degree is used to calculate the activation weight of each of the rules in the BRB to demonstrate its relative importance by using Equation 5 [65].

$$
\omega_{k}=\frac{\theta_{k} \alpha_{k}}{\sum_{i=1}^{L} \theta_{i} \alpha_{i}}
$$

where $\omega_{k}$ represents the rule activation weight, while $\alpha_{k}$ represents the combined matching degree, whereas $\theta_{k}$ represents the rule weight of the $k$ th rule.

If this activation weight of a rule becomes zero then the rule is said to be not activated or it has no impact.

\subsubsection{Belief Update}

The ignorance can be noticed when it is not possible to collect the input value of one of the antecedent attributes of a rule. In that case the belief degree embedded with the referential values of the consequent attributes during the development of the initial BRB should be updated and this can be achieved by applying Equation (6) [66]:

$$
\beta_{i k}=\bar{\beta}_{i k} \frac{\sum_{t=1}^{T_{k}}\left(\tau(t, k) \sum_{j=1}^{J_{t}} \alpha_{t j}\right)}{\sum_{t=1}^{T_{k}} \tau(t, k)}
$$

where $\alpha_{t j}$ is the matching degree of the $j$ th referential value of the $t$ th antecedent attribute as explained in Equation $3, \bar{\beta}_{i k}$ is the original belief degree, and $\beta_{i k}$ is the updated belief degree of the $i$ th referential value of the consequent attribute of the $k$ th rule, and

$$
\tau(t, k)=\left\{\begin{array}{l}
1, \text { if } P_{i} \text { is used in defining } R_{k}\left(t=1, \ldots, T_{k}\right) \\
0, \text { otherwise }
\end{array}\right.
$$

\subsubsection{Rule Aggregation}

In order to predict the PUE based on the input values of the antecedent attributes like external and room temperature, the activated rules need to be aggregated. The aggregation can be achieved by applying the Equation 7, which encapsulate the evidential reasoning algorithm [65] [66], where $\beta_{j}$ represents the aggregated final belief degree of the $j$ th referential value of the consequent.

When the prediction level of PUE is obtained using Equation 7 with certain belief degrees associated with each of the referential values (Very High, High, Medium, Low, Very Low), this can be converted into a crisp value, $y$, by using some preference values, $u$, with the help of Equation 8. This can be converted into a crisp value by assigning a utility score to each referential value of the consequent attribute with the help of Equation 8 where $y_{m}$ is the expected score expressed as a numerical value, $u\left(C_{n}\right)$ the utility score of each referential value $C_{n}$ of the consequent attribute, while $\beta_{n}$ its degree of belief.

$$
y_{m}=\sum_{n=1}^{N} u\left(C_{n}\right) * \beta_{n}
$$

\subsection{Optimal Learning Methods to Train the BRBES}

The learning parameters, including rule weights, attribute weights and consequent belief degrees $\left(\theta_{k}, \delta_{i}, \beta_{j k}\right)$ in a BRB can be acquired from domain experts or can be generated randomly. However, these parameters may not be 100\% accurate. This accuracy can be obtained by training the BRB using historical data [69]. Therefore, the aim of BRB training is to obtain an optimal set of parameters $\left(\theta_{k}, \delta_{i}, \beta_{j k}\right)$ that will minimize the discrepancy $\zeta(P)$ between the BRBES results $\left(y_{m}\right)$ and real system outputs $\left(\bar{y}_{m}\right)$, as shown in Figure 5. Several online and offline BRB training models can be found [69] [70] [71]. An optimization model for training the BRBES has been developed in this research where three important steps [72], consisting of (a) the construction of an objective function; (b) setting constraints for the training parameters; and (c) development of a training module to search for the optimal parameter set $\left(\theta_{k}, \delta_{i}, \beta_{j k}\right)$ have been considered, assuming there are $M$ cases in a training sample, and the inputoutput pairs of the $M$ cases are $\left(\bar{P}_{m}, \bar{y}_{m}\right)(m=1, \ldots, M)$.

\section{Belief Rule based Expert System to Pre- DICT PUE}

\subsection{Problem Description}

To reiterate, the dataset for this paper is obtained from the project funded under the JISC Greening ICT initiative (call for projects $14 / 10$ of October 2010) which was an experimental analysis providing real data relating to power measurement in different parts of a data center [1] [2]. The focus of this project was the impact of the temperature parameter on data energy efficiency (i.e. the PUE of the data center). The changes implemented included varying the AHU set point temperature between $21^{\circ} \mathrm{C}$ and $26^{\circ} \mathrm{C}$ in $2{ }^{\circ} \mathrm{C}$ steps, transferring the air cooling function to a more efficient free cooling system. An analysis of the average PUE for Leeds Beckett University (note: known as Leeds Metropolitan University prior to 2014) data centre was conducted for data collected from mid July 2012 till December 2012. The types of analyses conducted were constrained to trend analyses and comparative analyses. Thus, this paper aims to extend the original research work by incorporating machine learning and uncertainty into PUE analyses of the monitored data. However, our research is limited to the prediction of PUE by taking account of External Temperature (ExT) and Room Temperature (RT) due to the constrained nature of the monitored data for the stipulated period (note: our model could be easily extended to encompass other factors but subject to availability of data relating to humidity, fan speeds, speed of airflow, power density, etc.). Table 2 shows the process data where column 1 is related to external data, column 2 is related to room temperature data, while column 3 shows the actual PUE. We will use the data to train and validate a belief rule based expert system for 


$$
\beta_{j}=\frac{\mu \times\left[\prod_{k=1}^{L}\left(\omega_{k} \beta_{k j}+1-\omega_{k} \sum_{j=1}^{N} \beta_{k j}\right)-\prod_{k=1}^{L}\left(1-\omega_{k} \sum_{j=1}^{N} \beta_{k j}\right)\right]}{1-\mu \times\left[\prod_{k=1}^{L}\left(1-\omega_{k}\right)\right]}
$$

with

$\mu=\left[\sum_{j=1}^{N} \prod_{k=1}^{L}\left(\omega_{k} \beta_{k j}+1-\omega_{k} \sum_{j=1}^{N} \beta_{k j}\right)-(N-1) \times \prod_{k=1}^{L}\left(1-\omega_{k} \sum_{j=1}^{N} \beta_{k j}\right)\right]^{-1}$

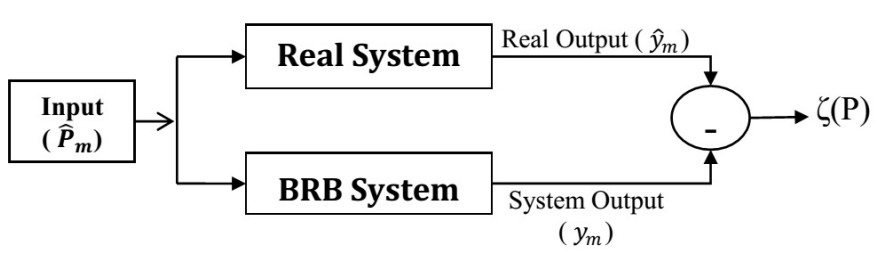

Fig. 5. Illustration of Optimal Learning Process.

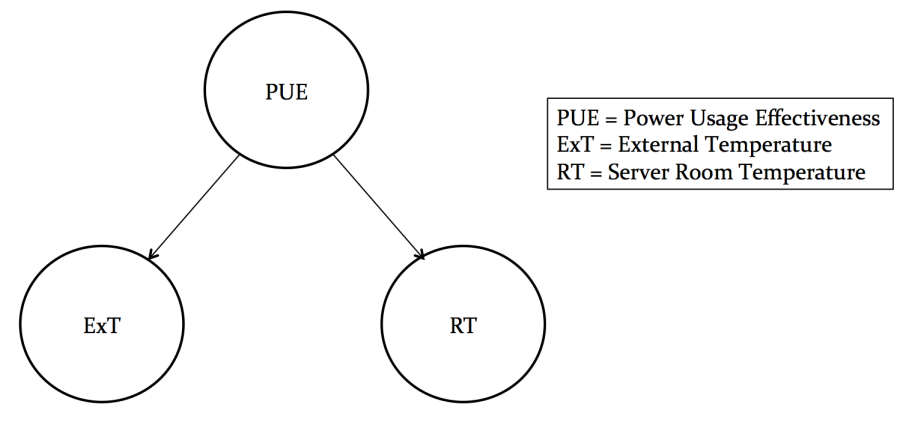

Fig. 6. BRB Framework to Predict PUE.

predicting PUE. By taking into account of the data shown in Table 2, a BRB framework has been developed as shown in Figure 6.

\subsection{Knowledge-base of the BRBES}

In order to construct the knowledge base for this expert system by using a belief rule base, a BRB framework has been developed, as illustrated in Figure 6. From the framework illustrated in Figure 6, it can be observed that input factors for determining PUE include External Temperature (ExT) and Room Temperature (RT); and hence this BRB has two antecedent attributes. The number of referential values considered for each antecedent attribute decide the size of the rule base. If the number is too large, there will be too many rules in the rule base, and the subsequent training and inference process will become more complicated. On the contrary, if it is too small the values may not be able to cover the range of antecedent attributes. Normally 5-9 referential values are used. The number of referential values for a consequent attribute is also comparable to those of the antecedent attributes. In this article, we use five referential values for External Temperature (ExT) with the following categorical values: "Very High", "High", "Medium", "Low", and "Very Low", and the five number of referential values with the same categories for the Room Temperature (RT) antecedent attribute. Hence, our BRB consists of 25 (i.e. $5^{2}$ ) rules. For the consequent attribute "PUE" we also use five
TABLE 2

Collected data

\begin{tabular}{|c|c|c|c|}
\hline Case No & $\begin{array}{c}\text { External } \\
\text { Temp. } \\
(\text { ExT })^{\circ} \mathrm{C}\end{array}$ & $\begin{array}{c}\text { Room } \\
\text { Temp. } \\
(\mathrm{RT}){ }^{\circ} \mathrm{C}\end{array}$ & PUE \\
\hline 1 & 12.2 & 24 & 1.211911611 \\
2 & 3.7 & 24 & 1.794087197 \\
3 & 3.7 & 26 & 1.812741602 \\
4 & 4.3 & 26 & 1.43041201 \\
5 & 5.1 & 24 & 1.755342547 \\
6 & 5.1 & 26 & 1.748201285 \\
7 & 5.1 & 24 & 1.729254114 \\
8 & 5.5 & 26 & 1.200023856 \\
9 & 5.5 & 21 & 0.891032363 \\
10 & 5.5 & 24 & 2.248577241 \\
11 & 5.5 & 26 & 1.797159206 \\
12 & 5.5 & 24 & 1.77400747 \\
13 & 6.1 & 26 & 1.425286882 \\
14 & 6.9 & 26 & 1.741909423 \\
15 & 8 & 26 & 1.857433678 \\
16 & 9 & 21 & 1.211413344 \\
17 & 9 & 24 & 1.213347098 \\
18 & 9 & 26 & 1.213868115 \\
19 & 9 & 26 & 1.556781265 \\
20 & 11.5 & 26 & 1.279506147 \\
21 & 12.2 & 21 & 1.219301368 \\
22 & 12.7 & 26 & 1.553739092 \\
23 & 15.3 & 26 & 1.474097426 \\
24 & 16.3 & 26 & 1.600396482 \\
25 & 13.4 & 26 & 1.346182288 \\
\hline & & & \\
\hline
\end{tabular}

referential values with the same categories. Collected data is shown in Table 2.

The referential values defined above for the antecedent and consequent attributes have categorical values which need to be further quantified. By examining the external temperature, the room temperature, and the recorded PUE value, the following relationship between the categorical values and numerical values are assumed so that the values approximately cover the corresponding attribute value range.

For the external temperature (ExT) it is assumed that 


$$
\text { ExT: }\left\{\begin{array}{l}
\text { Very High }(\mathrm{VH})-17^{\circ} \mathrm{C} \\
\text { High }(\mathrm{H})-13.5^{\circ} \mathrm{C} \\
\text { Medium }(\mathrm{M})-10^{\circ} \mathrm{C} \\
\text { Low }(\mathrm{L})-6.5^{\circ} \mathrm{C} \\
\text { Very Low }(\mathrm{VL})-3^{\circ} \mathrm{C}
\end{array}\right.
$$

TABLE 3

Initial Belief Rule Base for PUE

For the room temperature (RT), it is assumed that

$$
\text { RT: }\left\{\begin{array}{l}
\text { Very High }(\mathrm{VH})-26^{\circ} \mathrm{C} \\
\text { High }(\mathrm{H})-24.5^{\circ} \mathrm{C} \\
\text { Medium }(\mathrm{M})-23^{\circ} \mathrm{C} \\
\text { Low }(\mathrm{L})-21.5^{\circ} \mathrm{C} \\
\text { Very Low }(\mathrm{VL})-20^{\circ} \mathrm{C}
\end{array}\right.
$$

For PUE, it is assumed that

$$
\text { PUE: }\left\{\begin{array}{l}
\text { Very High }(\mathrm{VH})-2 \\
\text { High }(\mathrm{H})-1.75 \\
\text { Medium }(\mathrm{M})-1.5 \\
\text { Low }(\mathrm{L})-1.25 \\
\text { Very Low }(\mathrm{VL})-1
\end{array}\right.
$$

Using the categorical values and their respective numerical values, one of the conventional IF-THEN rules for PUE prediction could be like this:

IF ExT is Medium and RT is High THEN PUE is Medium However, such traditional IF-THEN rules are incapable of capturing continuous non-linear causal relationships between the antecedents and the consequent. Therefore, it is difficult to achieve the optimal performance of an expert system using those rules. Hence, by using the concept of a belief rule base, the traditional IF-THEN rule can be extended as follows:

IF ExT is Medium and RT is High THEN PUE is (VH, 0.6), (H, 0.1) (M, 0.1), (L, 0.1), (VL, 0.1)

A BRB can be established in four ways: 1) Extracting belief rules from expert knowledge; 2) Extracting belief rules by examining historical data; 3 ) Using the previous rule bases, if available; and 4) Creating random rules without any prior knowledge. In this article, an initial BRB has been constructed by taking into account of domain expert knowledge and is used as the starting point for training. Table 3 lists the initial 25 belief rules provided by an expert experienced in PUE detection. However, the belief degrees for PUE distribution (Table 3) may not be accurate. Therefore, further training is necessary to fine tune the belief degrees so that the reliability of the BRBES can be improved or optimised.

\subsection{Inference Engine using ER Approach}

The inference engine of this BRB expert system used the Evidential Reasoning (ER) algorithm [75], which is described in Section 3. The inference engine works as follows [76]: i) it first reads in input data (note: in this research they are external temperature and room temperature); ii) the

\begin{tabular}{|c|c|c|c|c|c|c|c|c|}
\hline & & \multicolumn{2}{|c|}{ IF } & \multicolumn{5}{|c|}{ THEN } \\
\cline { 5 - 9 } Rule & Rule & ExT & RT & \multicolumn{5}{|c|}{ PUE } \\
\cline { 5 - 9 } Id & Weight & & & VH & H & M & L & VL \\
& & & & & & & & \\
\hline R1 & 1.0 & VH & VH & 1.00 & 0.00 & 0.00 & 0.00 & 0.00 \\
R2 & 1.0 & VH & H & 0.50 & 0.50 & 0.00 & 0.00 & 0.00 \\
R3 & 1.0 & VH & M & 0.50 & 0.00 & 0.50 & 0.00 & 0.00 \\
R4 & 1.0 & VH & L & 0.50 & 0.00 & 0.00 & 0.50 & 0.00 \\
R5 & 1.0 & VH & VL & 0.50 & 0.00 & 0.00 & 0.00 & 0.50 \\
R6 & 1.0 & H & VH & 0.50 & 0.50 & 0.00 & 0.00 & 0.00 \\
R7 & 1.0 & H & H & 0.00 & 1.00 & 0.00 & 0.00 & 0.00 \\
R8 & 1.0 & H & M & 0.00 & 0.50 & 0.50 & 0.00 & 0.00 \\
R9 & 1.0 & H & L & 0.00 & 0.50 & 0.00 & 0.50 & 0.00 \\
R10 & 1.0 & H & VL & 0.00 & 0.50 & 0.00 & 0.00 & 0.50 \\
R11 & 1.0 & M & VH & 0.50 & 0.00 & 0.50 & 0.00 & 0.00 \\
R12 & 1.0 & M & H & 0.00 & 0.50 & 0.50 & 0.00 & 0.00 \\
R13 & 1.0 & M & M & 0.00 & 0.00 & 1.00 & 0.00 & 0.00 \\
R14 & 1.0 & M & L & 0.00 & 0.00 & 0.50 & 0.50 & 0.00 \\
R15 & 1.0 & M & VL & 0.00 & 0.00 & 0.50 & 0.00 & 0.50 \\
R16 & 1.0 & L & VH & 0.50 & 0.00 & 0.00 & 0.50 & 0.00 \\
R17 & 1.0 & L & H & 0.00 & 0.50 & 0.00 & 0.50 & 0.00 \\
R18 & 1.0 & L & M & 0.00 & 0.00 & 0.50 & 0.50 & 0.00 \\
R19 & 1.0 & L & L & 0.00 & 0.00 & 0.00 & 1.00 & 0.00 \\
R20 & 1.0 & L & VL & 0.00 & 0.00 & 0.00 & 0.50 & 0.50 \\
R21 & 1.0 & VL & VH & 0.50 & 0.00 & 0.00 & 0.00 & 0.50 \\
R22 & 1.0 & VL & H & 0.00 & 0.50 & 0.00 & 0.00 & 0.50 \\
R23 & 1.0 & VL & M & 0.00 & 0.00 & 0.50 & 0.00 & 0.50 \\
R24 & 1.0 & VL & L & 0.00 & 0.00 & 0.00 & 0.50 & 0.50 \\
R25 & 1.0 & VL & VL & 0.00 & 0.00 & 0.00 & 0.00 & 1.00 \\
\hline
\end{tabular}

input data are then transformed by using Equation 3; iii) it calculates the activation weight of all rules using Equation 5 ; iv) it updates the belief degree of consequence in rules using Equation 6; and v) finally, it aggregates all rules by using Equation 7.

\subsection{Training the Rule Base}

25 sets of data are considered and hence the value of $M$ is 25.

\subsubsection{Training}

The BRBES optimal training model is briefly introduced in Section 3.3. The objective of the learning process is to find a set of training parameters, $P$, so that the difference between the observed $\left(y_{m}\right)$ and the estimated PUE is minimized as illustrated in Figure 5 as well as in Equation 13.

$$
\zeta(P)=\frac{1}{M} \sum_{m=1}^{M}\left(y_{m}-\bar{y}_{m}\right)^{2}
$$

The simulated or estimated $\operatorname{PUE}\left(\hat{y}_{m}\right)$ for certain input data can be obtained by following the procedures mentioned in Section 4.2.

Four different sets of training parameters have been considered for training the BRB as given below, where the same objective function (Equation 13) for each training round is used. 
T1: Training with different sets of proportional and reciprocal referential values of the two antecedent attributes, namely external temperature and room temperature.

T2: Training with different sets of rule weight

T3: Training with different sets of attribute weight

T4: Training with referential values of the antecedent attributes, rule weight $\left(\theta_{k}\right)$, antecedent attribute weight $\left(\delta_{k}\right)$, consequent belief degrees $\left(\beta_{j k}(j=1, \ldots, 5, k=1, \ldots, L)\right)$. In this case, the best values of the parameters including referential values of the antecedent attribute, antecedent attribute weight and rule weight, which generate the minimum errors are considered along with consequent belief degrees.

The following constraints and initial value for each parameter have been considered.

(1) Severity scores of the five referential levels $\mu\left(C_{j}\right)(j=1, \ldots, 5)$

$2 \geq \mu\left(C_{j}\right)(j=1, \ldots, 5) \geq 1$;

$\mu\left(C_{1}\right.$ (Very High $\left.)\right) \geq \mu\left(C_{2}\right.$ (High $\left.)\right) \geq$

$\geq \mu\left(C_{3}(\right.$ Medium $\left.)\right) \geq \mu\left(C_{4}(\right.$ Low $\left.)\right) \geq \mu\left(C_{5}\right.$ (Very Low $\left.)\right)$;

$\mu\left(C_{1}\right.$ (Very High $\left.)\right)=2.0$,

$\mu\left(C_{2}\right.$ (High $\left.)\right)=1.75$,

$\mu\left(C_{3}(\right.$ Medium $\left.)\right)=1.5$,

$\mu\left(C_{4}(\right.$ Low $\left.)\right)=1.25$,

$\mu\left(C_{5}\right.$ (Very Low $\left.)\right)=1.0$

The range of the utility value for the antecedent attribute External Temperature has been maintained between 3-17 since this is the range value obtained from the dataset as shown in Table 2. On the other hand, the range of the utility value for the antecedent attribute Room Temperature (RT) has been kept between 20-26 since this is the range value obtained from the dataset as shown in Table 2.

(2) Rule weights $\theta_{k}(k=1, \ldots, L)$ :

$1 \geq \theta_{k}(k=1, ., L) \geq 0$

$\theta_{k}(k=1, ., L)=1$;

(3) Antecedent attribute weights $\delta_{k}, k \in 1, \ldots, L$ :

$1 \geq \delta_{k}(k=1, ., L) \geq 0$

$\delta_{k}(k=1, ., L)=1$; $L)$ :

(4) Consequent belief degrees $\beta_{j k}(j=1,2,3,4,5, k=1, \ldots$,

$$
\begin{aligned}
& 1 \geq \beta_{j k}(j=1, \ldots, 5, k=1, \ldots, L) \geq 0, \text { and } \\
& 1 \geq \sum_{j=1}^{5} \beta_{j k}(k=1, \ldots, L) \geq 0
\end{aligned}
$$

The final step of the training includes obtaining the optimal value of the training parameters. Random numbers generated by the random function of the math class from java. lang have been used by considering the above constraints for each of the four different set of training parameters. Hence, the output of the training consists of acquiring the set of optimal parameter values which will reduce the uncertainty associated with the initial rule base. In this way, the optimal values of the parameters have been obtained.

The trained belief rules are listed in Table 5 . The difference between the observed and the estimated PUE obtained using BRBES as defined in Equation 13 is 0.043328427.

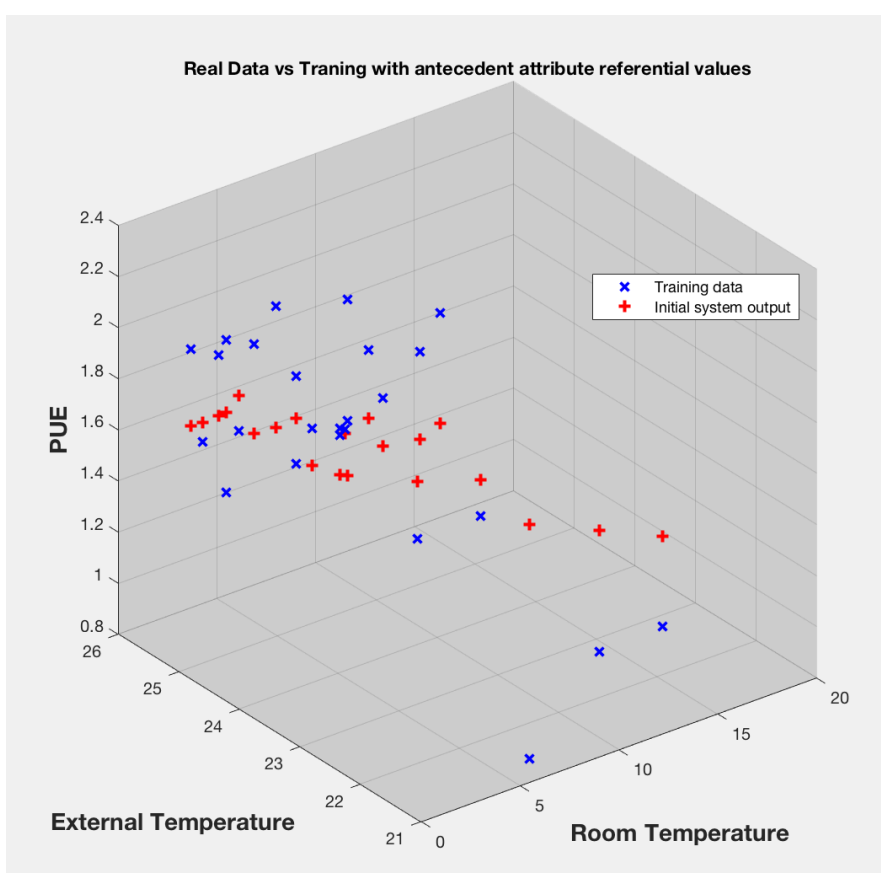

Fig. 7. Real Data vs. Training with Antecedent Attribute Referential Values.

\section{Results and Discussion}

$\mathrm{T}$ HE estimated PUE calculated using Equation 8 based on the initial belief rule base is plotted in Figure 7 together with the observed PUE in the training dataset. It is obvious that the estimated values do not match the observed values very well. This means the initial rule base provided by an expert is not good enough. The error obtained by applying Equation 13 stands at 0.1313. The Receiver Operating Characteristics (ROC) curve is widely used to analyze the effectiveness of assessment having ordinal or continuous results [72]. Therefore, the method was considered, to measure the reliability of BRBES in comparison with the real system output i.e. the data center in Leeds. The reliability of the BRBES can be measured by calculating the Area Under Curve (AUC) [73] [74]. The larger the AUC, the higher is the accuracy and reliability of results. Figure 8 shows the ROC curve. The ROC curve with red line in Figure 8 illustrates the BRBES result while the curve with blue line illustrates the real system result. The AUC for BRBES is 0.551 (95\% confidence intervals 0.695 0.930), and the AUC of real system is 1 (95\% confidence intervals 0.651 0.907). From the AUC of the BRBES results and real system results, it can be observed that AUC of BRBES is less than that of the AUC of real system. This indicates that the results generated by the BRBES need to be improved to obtain an accurate prediction value of PUE against certain external and room temperatures. SPSS 20.0 has been used to construct the ROC curve and to calculate the AUC of these curves.

Therefore, to increase the prediction accuracy of the BRBES, an optimal learning training module is developed. The different combinations of training parameters (T1, T2, $\mathrm{T} 3, \mathrm{~T} 4)$ are considered. While training with different combinations of the antecedent attributes (T1) is carried out, it appears that the reciprocal combination of the referential values which is high to low and low to high, generates 


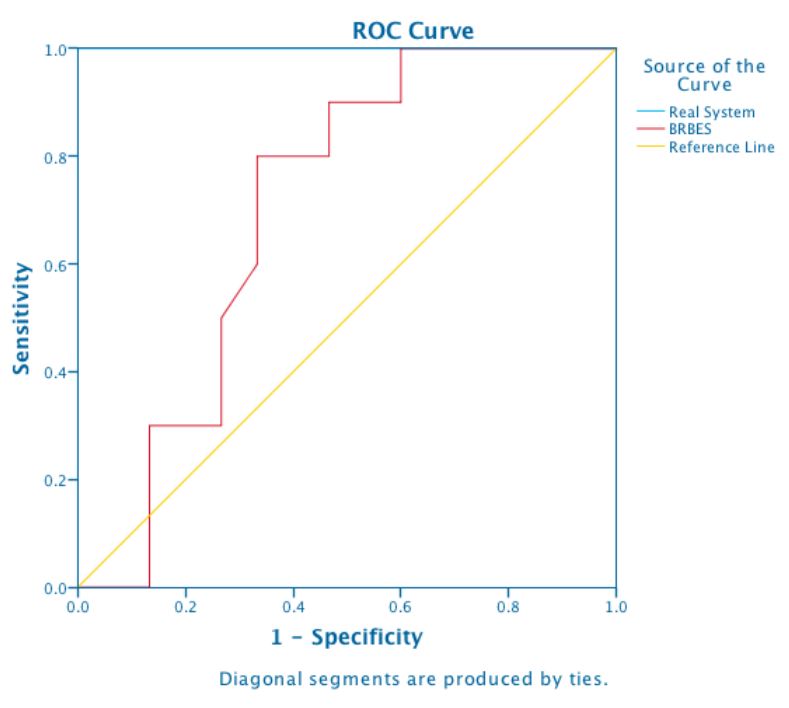

Fig. 8. BRBES's ROC for random initial BRB.

TABLE 4

Comparison of results among training parameters.

\begin{tabular}{|c|c|c|}
\hline Training Parameter & AUC of ROC & Estimated error \\
\hline T11 (H-L, H-L) & 0.551 & 0.1313 \\
T12 (L-H, H-L) & 0.333 & 0.186098 \\
T13 (L-H, L-H) & 0.474 & 0.10328 \\
T14 (H-L, L-H) & 0.679 & 0.083946 \\
T2 & 0.474 & 0.10328 \\
T3 & 0.468 & 0.104544 \\
T4 & 0.854 & 0.043328427 \\
ANN & 0.55 & 0.0521 \\
GA1 (Rule Weight) & 0.74 & 0.0461 \\
GA2 (Attribute Weight) & 0.65 & 0.0815 \\
\hline
\end{tabular}

better accuracy in prediction. The estimated error in this case, stands at 0.083946, which is less than the estimated error found in the BRBES without training, which is 0.1313 . Therefore, it is evident the degree of match for the observed and estimated values in Figure 9 is better compared to Figure 7. Table 4 illustrates the comparison of ROC values and estimated errors against different combinations of referential values (T1). The value for the AUC as well as estimated errors for other training parameters (T2, T3, T4) can be found in Table 4. It can be observed that the training parameter $\mathrm{T} 4$ is producing the best result since the estimated error is now reduced to 0.043328427 . This is also evident from obtaining the greater AUC value which is 0.854 and hence much closer to one, i.e to the real system. The reason for a better performance of the training parameter $\mathrm{T} 4$ is that it combines the best parameters from T1 (which is reciprocal approach of antecedent attribute referential values), T2 (Attribute Weight), T3 (Rule Weight) as well as a chosen optimal degree of beliefs for the 25 rules. 4000 random values have been generated using the java. lang library function out of these values, an optimal value is chosen. Consequently, the trained belief rule base is developed as shown in Table 5.

In addition to the above optimal learning methods,

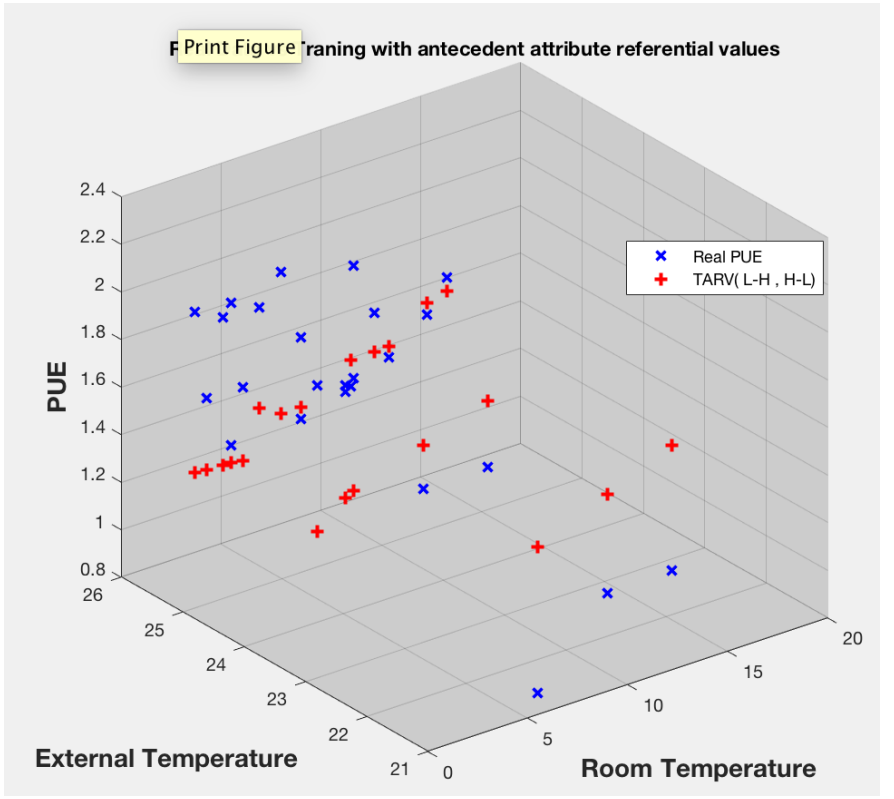

Fig. 9. Real Data vs. Training with Antecendent Attribute Referential Values.

TABLE 5

Trained BRB for PUE

\begin{tabular}{|c|l|c|c|c|c|c|c|c|}
\hline & & \multicolumn{2}{|c|}{ IF } & \multicolumn{5}{|c|}{ THEN } \\
\cline { 5 - 9 } Rule & Rule & ExT & RT & \multicolumn{5}{|c|}{ PUE } \\
\cline { 5 - 9 } Id & Weight & & & VH & H & M & L & VL \\
& & & & & & & & \\
\hline R1 & 1.0 & VH & VH & 0.262 & 0.176 & 0.259 & 0.188 & 0.115 \\
R2 & 1.0 & VH & H & 0.203 & 0.249 & 0.000 & 0.109 & 0.439 \\
R3 & 1.0 & VH & M & 0.281 & 0.037 & 0.044 & 0.181 & 0.458 \\
R4 & 1.0 & VH & L & 0.239 & 0.026 & 0.197 & 0.168 & 0.370 \\
R5 & 1.0 & VH & VL & 0.141 & 0.050 & 0.231 & 0.151 & 0.427 \\
R6 & 1.0 & H & VH & 0.198 & 0.225 & 0.178 & 0.108 & 0.291 \\
R7 & 1.0 & H & H & 0.184 & 0.261 & 0.263 & 0.162 & 0.130 \\
R8 & 1.0 & H & M & 0.087 & 0.079 & 0.029 & 0.101 & 0.705 \\
R9 & 1.0 & H & L & 0.020 & 0.170 & 0.067 & 0.149 & 0.593 \\
R10 & 1.0 & H & VL & 0.067 & 0.168 & 0.024 & 0.183 & 0.559 \\
R11 & 1.0 & M & VH & 0.111 & 0.235 & 0.252 & 0.031 & 0.371 \\
R12 & 1.0 & M & H & 0.206 & 0.084 & 0.072 & 0.037 & 0.601 \\
R13 & 1.0 & M & M & 0.010 & 0.099 & 0.086 & 0.193 & 0.613 \\
R14 & 1.0 & M & L & 0.099 & 0.132 & 0.034 & 0.229 & 0.506 \\
R15 & 1.0 & M & VL & 0.057 & 0.263 & 0.144 & 0.086 & 0.450 \\
R16 & 1.0 & L & VH & 0.106 & 0.141 & 0.269 & 0.174 & 0.310 \\
R17 & 1.0 & L & H & 0.251 & 0.162 & 0.145 & 0.116 & 0.327 \\
R18 & 1.0 & L & M & 0.110 & 0.186 & 0.256 & 0.092 & 0.356 \\
R19 & 1.0 & L & L & 0.029 & 0.172 & 0.241 & 0.207 & 0.351 \\
R20 & 1.0 & L & VL & 0.272 & 0.088 & 0.048 & 0.115 & 0.476 \\
R21 & 1.0 & VL & VH & 0.216 & 0.185 & 0.016 & 0.084 & 0.499 \\
R22 & 1.0 & VL & H & 0.232 & 0.268 & 0.053 & 0.015 & 0.432 \\
R23 & 1.0 & VL & M & 0.255 & 0.282 & 0.276 & 0.077 & 0.109 \\
R24 & 1.0 & VL & L & 0.154 & 0.271 & 0.012 & 0.241 & 0.322 \\
R25 & 1.0 & VL & VL & 0.100 & 0.073 & 0.091 & 0.023 & 0.713 \\
\hline
\end{tabular}

Genetic Algorithm (GA) based learning approach is employed using the trained belief degrees. The rule weights and the attribute weights are trained using GA. It can be 


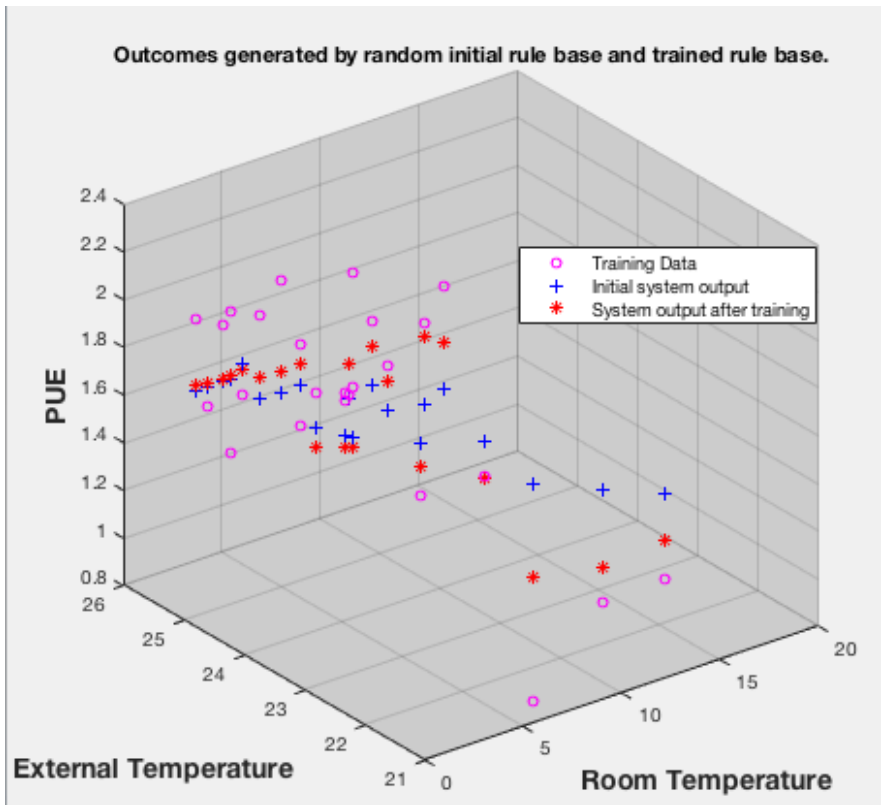

Fig. 10. Outcomes generated by random initial rule base and trained rule base.

observed from Table 4 that the error in rule weight [GA1 (Rule Weight)] is less than that of the attribute weight [GA2 (Attribute Weight)]. However, the error is not less than that of T4. The reason for this is that the different types of uncertainties have been considered in the BRBES inference process, while this is not the case with GA. In addition, an Artificial Neural Network (ANN) is used to develop an optimal learning model. It comprises a feed forward architecture and 10 hidden layers with a back propagation learning algorithm. Table 4 shows that the error in the ANN is higher than that of T4. The reason for this is that in the neural network only one learning parameter is considered while in the BRBES approach, many learning parameters such as belief degree, rule weight and attribute weight are considered.

Figure 10 illustrates the comparison between the results generated by the random initial belief rule base and the trained belief rule base. It can be observed from Figure 10 that the degree of match for the trained BRB and observed data is much closer. In this way, an optimal learning model developed for this research fosters a more accurate prediction of the datacenter PUE using external and internal room temperatues.

\section{CONCLUSION AND FutURE WORK}

$I^{r}$ $\mathrm{N}$ conclusion, this article has demonstrated how BRBES could be employed for predictive modeling with uncertainty. It seems to have been successfully deployed for the prediction (with uncertainty) of energy efficiency of a data center. In this research, four different sets of training parameters have been administered onto the data center energy efficiency dataset. To reiterate, they are: T1 - training with different sets of proportional and reciprocal referential values of two antecedent attributes, namely external temperature and room temperature; T2 - training with different sets of rule weight; T3 - training with different sets of attribute weight; T4 - training with referential values of the antecedent attributes, rule weight, antecedent attribute weight, and consequent belief degrees. The training parameter T4, has yielded the best results with an estimated error of only 0.043328427 and AUC value of 0.854 (note: this is very close to 1 , the AUC value for the real system). These promising results are due to the fact that $\mathrm{T} 4$ is an integration of all the appropriate parameters from $\mathrm{T} 1, \mathrm{~T} 2$, and $\mathrm{T} 3$ as well as the optimal degree of belief considered for all the 25 rules. The proposed optimal learning algorithm performs better than that of ANN (Artificial Neural Network) and GA (Genetic Algorithm) (as can be seen from Table 4). The reason for this is that various types of uncertainties have been considered in the inference process of BRBES, which is neither the case for ANN, nor GA. Moreover, ANN considers less number of learning parameters than BRBES.

The BRB framework discussed in this article could be further extended to incorporate other energy efficiency factors discussed in Section 2 of this paper: humidity, power density, fan speeds, rate of airflow, etc. Furthermore, we intend to validate our model in a real-world setting.

\section{ACKNOWLEDGMENTS}

The authors record their thanks to the JISC Greening ICT project for funding the work on data center energy efficiency. We also record our appreciation of technical colleagues at Leeds Beckett University for their technical support throughout the project. Last, but not least, the authors record their thanks to the Swedish Research Council granting (Grant 2014-4251) financial support.

\section{References}

[1] C. Pattinson and R. Cross, "Measuring Data Centre Efficiency," JISC Green IT Technical Report. ISBN: 978-1-907240-33-1, 2013.

[2] C. Pattinson, A. Kor, and R. Cross, "Critical Issues for Data Center Energy Efficiency," Green Information Technology: A Sustainable Approach. Elsevier. ISBN 978-0-12-801379-3, 2015.

[3] A. Shehabi, S. Smith, D. Sartor, R. Brown, M. Herrlin, J. Koomey, E. M. N. Horner, I. Azevedo,and W. Lintner, "United States Data Center Energy Usage Report," Ernest Orlando Lawrence Berkeley National Laboratory, Berkeley, CA, USA, LBNL-1005775, 2016. URL: https://publications.lbl.gov/islandora/object/ir\%3A1005775/ datastream/PDF/view

[4] F. Palmieri, S. Ricciardi, U. Fiore, M. Ficco, and A. Castiglione, "Energy-oriented denial of service attacks: an emerging menace for large cloud infrastructures,", J. Supercomput. (2015) 71:1620

[5] Microsoft, "Best Practices For Energy Efficiency In Microsoft Data Center Operations," Factsheet, 2008, URL: http:/ / download.microsoft.com/download/a/7/b/a7b72ab1ca17-4589-923a-83b0ff57be6d/energy-efficiency-best-practices-inmicrosoft-data-center-operations-cebit.doc

[6] [online] https://www.datacenterjournal.com/data-center-energyconsumption-context

[7] [online] http://www.independent.co.uk/environment/globalwarming-data-centres-to-consume-three-times-as-much-energy-innext-decade-experts-warn-a6830086.html

[8] [online] http://www.computerworld.com/article/2598562/datacenter/data-centers-are-the-new-polluters.html

[9] [online] http://datacenterfrontier.com/report-data-centers-areenergy-misers-not-power-hogs/

[10] [online] http://www.dell.com/learn/us/en/uscorp1/dellenvironment-energy-efficiency

[11] [online] https://energy.gov/sites/prod/files/2013/10/f3/eedatacenterbestpractices.pdf

[12] [online] https://www.energystar.gov/products/low_carbon_it_campaign/12_ways_save_energy_data_center 
[13] [online] https://www.energystar.gov/products/low_carbon_it_campaign/12_ways_save_energy_data_center/purchasing_more_energy_efficient_servers_upss_and_pdus

[14] [online] http://gartnerinfo.com/futureofit2011/MEX38L_B4\%20mex381_b4.pdf

[15] [online] http://searchdatacenter.techtarget.com/definition/serverconsolidation

[16] [online] https://www.vmware.com/uk/solutions/consolidation.html

[17] [online] http://blu.org/meetings/2002/12/paper.pdf

[18] [online] http://download.microsoft.com/download/A/E/8/AE8277FA-205D-49D5-8224-D31F5771779B/Datacenter-

Consolidation-and-Migration-Datasheet.pdf

[19] [online] http://www-935.ibm.com/services/in/gts/pdf/serverconsolidation-data-sheet-final-041207.pdf

[20] [online] http://searchdatacenter.techtarget.com/definition/serverconsolidation

[21] [online] https://technet.microsoft.com/enus/library/ee819082(v=sql.100).aspx

[22] [online] http://www.thegreengrid.org/ /media/WhitePapers/ White $\% 20$ Paper $\% 2019 \% 20-\% 20$ Using $\% 20$ Virtualization $\% 20$ to $\% 20$ Improve\%20Data\%20Center\%20Efficiency.pdf

[23] [online] https://www.vmware.com/content/dam/digitalmarketing/ vmware/en/pdf/vmware-reduce-power-consumption-wp.pdf

[24] [online] http://www.cisco.com/c/en/us/solutions/collateral/datacenter-virtualization/net_implementation_white per0900aecd806a9c05.html

[25] [online] https://www.energystar.gov/products/low_carbon_it_ campaign/12_ways_save_energy_data_center/server_virtualization

[26] [online] http://www.cisco.com/c/dam/en_us/about/ciscoitatwork/ downloads/ciscoitatwork/pdf/Cisco_IT_Case_Study_VMWare.pdf

[27] [online] http://www-05.ibm.com/za/event/previous/DI/ Virtualization.pdf

[28] [online] http://www.gartner.com/it/initiatives/pdf/KeyInitiative Overview Virtualization.pdf

[29] [online] http://www.thegreengrid.org/ $\sim /$ media/WhitePapers White_Paper_27_Impact_of_Virtualization_Data_On_Center_Physical Infrastructure 020210.pdf

[30] [online] http://www.datacenterknowledge.com/archives/2012/09/ 18/factors-that-impact-data-center-energy-efficiency /

[31] [online] http://www.criticalpowerandcooling.com/whitepapers/Cooling/WP-120\%20Guidelines $\% 20$ for $\% 20$ Specification $\% 20$ of_\%20Data $\% 20$ Center\%20Power\%20Density.pdf

[32] [online] http://www.computerweekly.com/tip/How-to-buildand- manage-an-energy-efficient-data-centre-A-mini-guide

[33] [online] http://www.nrel.gov/docs/fy14osti/61925.pdf

[34] [online] http://electrical-engineering-portal.com/single-phasepower-vs- three-phase-power

[35] [online] http://viewer.media.bitpipe.com/979246117_954/ 1296228818955/SchneiderElectricsDataCenterSO033163EGuide 012611.pdf

[36] [online] https://datacenters.lbl.gov/tools/5-data-center-airmanagement-tool-featured

[37] [online] http://www.nrel.gov/docs/fy11osti/47201.pdf

[38] [online] https://www.energystar.gov/products/low_carbon_it_ campaign/12_ways_save_energy_data_center/variable_speed_fan_drives

[39] [online] https://datacenters.lbl.gov/sites/all/files/dc_ fancasestudy.pdf 35.

[40] [online] https://www.energystar.gov/products/low_carbon_it_ campaign/12_ways_save_energy_data_center/containment_enclosure

[41] [online] http://www.on365.co.uk/files/Impact $\% 20$ of $\% 20$ Hot $\% 20$ and $\% 20$ Cold $\% 20$ Aisle.pdf

[42] [online] http://searchconvergedinfrastructure.techtarget.com/definition/data-center-infrastructure-management-DCIM

[43] [online] https://datacenters.lbl.gov/

[44] [online] http://ec.europa.eu/information_society/activities/sustaina _growth/docs/datacenter_code-conduct.pdf

[45] [online] http://www.cencenelec.eu/standards/sectors/ict/pages/ greendatacentres.aspx

[46] [online] https://www.energystar.gov/products/low_carbon_it _campaign/12_ways_save_energy_data_center

[47] [online] http://www.datacenterknowledge.com/archives/2015/04/21/ microsofts-seven-tenets-of-data-center-efficiency/

[48] [online] https://www.google.co.uk/about/datacenters/efficiency/
[49] [online] http://download.microsoft.com/download/1/1/9/ 119CD765-0CEE-4DA6-B396-20603D3F4701/Datacenter_Sustainability_ Strategy_Brief.pdf

[50] [online] https://www.energystar.gov/sites/default/files/asset/ document/Google_Server_Room_Case_Study_0.pdf

[51] [online] https://datacenters.lbl.gov/sites/all/files/WP49PUE\%20A\%20 Comprehensive \%20Examination\%20of \%20the $\% 20$ Metric_v6.pdf

[52] [online] https://www.google.co.uk/green/efficiency/datacenters/

[53] [online] http://www.bcs.org/upload/pdf/data-centreenergy.pdf

[54] [online] http://www.thermalnews.com/main/articles/predictivemodeling-the-next-frontier-in-data-center-condition-maintenance /

[55] [online] http://www.datacenterdynamics.com/contenttracks/serversfacility/83192.article

[56] [online] https://www.theguardian.com/environment/2016/jul/20/' google-ai-cut-data-centre-energy-use-15-per-cent

[57] [online] http://www.zdnet.com/article/predictive-modelingif-the- prognose-tools-really-work-the-will-become-datacenteressentials/

[58] [online] https://buildings.lbl.gov/sites/all/files/demo_of_data_ center_software.pdf

[59] [online] https://eetd.lbl.gov/publications/demonstration-ofdata-center- energy-u

[60] [online] https://www.google.co.jp/ja/jp/about/datacenters/ efficiency/internal/assets/machine-learning-applicationsfordatacenter- optimization-finalv2.pdf

61] M. K. Patterson, "The effect of data center temperature on energy efficiency," The 11th IEEE Intersociety Conference on Thermal and Thermomechanical Phenomena in Electronic Systems (ITHERM 2008), 2008.

[62] H. Coles, "Demonstration of Data Center Energy Use Prediction Software," Lawrence Berkeley National Laboratory, Berkeley, California. LBNL-6608E, 2013. URL: https://buildings.lbl.gov/sites/all/files/demo_of_data_center_ software.pdf

[63] R. Ul Islam, K. Andersson, and M. S. Hossain, "A Web Based Belief Rule Based Expert System to Predict Flood," Proceedings of the 17th International Conference on Information Integration and Web-based Applications \& Services (iiWAS 2015), 11-13 December 2015, Brussels, Belgium.

[64] M. S. Hossain, I. B. Habib, and K. Andersson, "A Belief Rule Based Expert System to Diagnose Dengue Fever Under Uncertainty," Accepted for Publication in the IEEE Technically Sponsored SAI Intelligent Conference 2017, July, London, UK.

[65] M. S. Hossain, P. O. Zander, S. Kamal, and L. Chowdhury, "A Belief Rule Based Expert System to Evaluate E-Government," Expert Systems: The Journal of Knowledge Engineering 32(5), John Wiley \& Sons Ltd, 2015.

[66] J.-B. Yang, J. Liu, J. Wang, H.-S. Sii, and H.-W. Wang, "Belief rule-base inference methodology using the evidential reasoning approach-rimer," IEEE Transactions on Systems, Man, and Cybernetics-part A: Systems and Humans 36(2):266-285, 2006.

[67] R. Ul Islam, M. S. Hossain, and K. Andersson, "A Novel Anomaly Detection Algorithm for Sensor Data Under Uncertainty," Soft Computing, Springer, DOI: 10.1007/s00500-016-2425-2, 2016.

[68] M. S. Hossain, M. A. Haque, R. Mustafa, R. Karim, H. R. Dey, and M. Yousuf, "An Expert System to Assist the Diagnosis of Ischemic Heart Disease," International Journal of Integrated Care, 2016.

69] J. B. Yang, J. Liu, D. L. Xu, J. Wang, and H. W. Wang, "Optimal learning method for training belief rule based systems, " IEEE Transactions on Systems, Man, and Cybernetics (Part A) 37:569-585, 2007.

70] Z. J. Zhou, C. H. Hu, J. B. Yang, D. L. Xu, and D. H. Zhou, "Online updating belief rule based system for pipeline leak detection under expert intervention," Expert Systems with Applications 36:77007709, 2009.

[71] Z. J. Zhou, C. H. Hu, J. B. Yang, D. L. Xu, M. Y. Chen, and D. H. Zhou, "A sequential learning algorithm for online constructing belief-rule-based systems," Expert Systems with Applications 37:1790-1799, 2010.

[72] Y. Yuan, S. Feldhamer, A. Gafni, F. Fyfe, and D. Ludwin, "The development and evaluation of a fuzzy logic expert system for renal transplantation assignment: is this a useful tool ${ }^{\prime}$ Euro. J. Oper. Res. 142:152-173, 2002. 
[73] C. E. Metz, "Basic principles of ROC analysis," Semin. Nucl. Med. 8(4):283-298, 1978.

[74] J. A. Hanley, "The Robustness of the Binormal Assumptions Used in Fitting ROC Curves," Med. Decis. Making 8(3):197-203, 1988.

[75] J. B. Yang, and P. Sen, "A general multi-level evaluation process for hybrid MADM with uncertainty," IEEE Transactions on Systems, Man and Cybernetics 24:1458-1473, 1994.

[76] D. L. Xu, J. Liu, J. B. Yang, G. P. Liu, J. Wang, I. Jenkinson, and J. Ren, "Inference and learning methodology of belief-rule-based expert system for pipeline leak detection," Expert Systems with Applications 32:103-113, 2007.

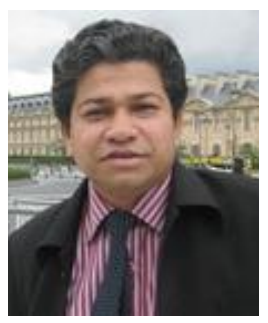

Mohammad Shahadat Hossain is a Professor of Computer Science and Engineering at the Chittagong University (CU), Bangladesh. He did both his MPhil and PhD in Computation from the University of Manchester Institute of Science and Technology (UMIST), UK in 1999 and 2002 respectively. His current research areas include e-government, the modeling of risks and uncertainties using evolutionary computing techniques. Investigation of pragmatic software development tools and methods, for information systems in general and for expert systems in particular are also his areas of research.

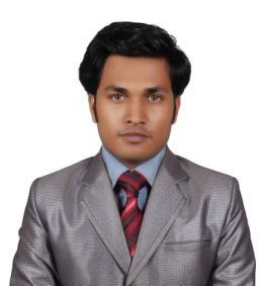

Saifur Rahaman is Lecturer of Computer Science \& Engineering at International Islamic University Chittagong, studying at masters level and was also a Research Assistant at the Computer Science \& Engineering Department at the University of Chittagong, Bangladesh until 2015 . He has published papers on Expert Systems and Biomedical Engineering. His current research interests include new techniques, tools, and methods to handle uncertainty for expert systems, biomedical engineering, clinical decision support systems, decision making and risk analysis in prediction models.

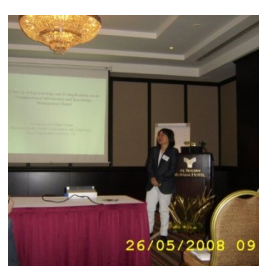

Ah-Lian Kor is a Course Leader of Leeds Beckett M.Sc. Sustainable Computing, specialising in software development, web applications, data analytics, and Al. She is active in sustainable IT, intelligent systems, decision support systems, and data center research. She forges an industrial collaboration with Drax Power Station which aims to develop and evaluate a virtual environment (in the form of an expert system) to support decision making in fault diagnosis for the centrifuge system for Drax Power Station. She is an Intelligent System Academic Expert for the Knowledge Transfer Partnership Project with Premier Farnell (a global electronics engineering company). Ah Lian is a Project Team Committee Member for several EU projects (e.g. ALIOT, GreenCo, PERCCOM, etc.). She has chaired sesssions in reputable international conferences (e.g. FTC 2016), a publicity chair for CyberSciTech 2017, sits in Technical Programme Committee for journals and conferences (e.g. ICAART, SEEDS, IARIA Intelligent Systems, IEEE Computing Conference, etc.).

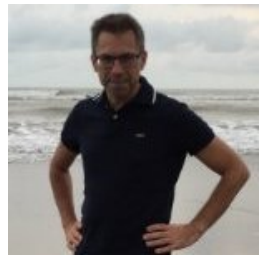

Karl Andersson has a M.Sc. degree in Computer Science and Technology from Royal Institute of Technology, Stockholm, Sweden and a Ph.D. degree in Mobile Systems from Luleå University of Technology, Sweden. After pursuing postdoctoral research at the Internet Real-time Laboratory at Columbia University, New York, USA and National Institute of Information and Communications Technology, Tokyo, Japan, he is now Associate Professor of Pervasive and Mobile Computing at Luleå University of Technology, Sweden. His research interests include Green and Mobile Computing, the Internet of Things, Cloud Technologies, and Information Security. 


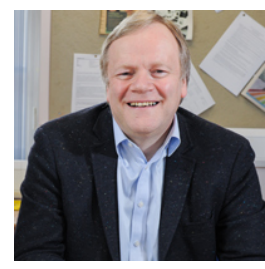

Colin Pattinson is Dean of the School of Computing, Creative Technologies and Engineering at Leeds Beckett University, UK. His research expertise includes: the relationship between power-saving network designs and management systems, low-cost, low-energy networking solutions. He is a member of the EU e-InfraNet Green ICT Network which aims to identify and promote an environmentally sustainable e-infrastructure for research and higher education in the ERA. He is leading projects on green computing technologies, and energy efficient data centers. He has recently completed a review for Cisco Systems of their energy monitoring and control software. 